\title{
Hidratación en el síndrome urémico hemolítico
} Hydration in hemolytic uremic syndrome

\author{
Dr. Manuel D. Bilkis ${ }^{a}$ y Dr. Pablo Bonany ${ }^{b}$
}

\section{RESUMEN}

El síndrome urémico hemolítico asociado a diarrea es precedido por una gastroenteritis por Escherichia coli productora de toxina Shiga. Se recomiendan medidas de sostén, especialmente, la restricción hídrica para evitar la sobrecarga cardiopulmonar. Sin embargo, la expansión de volumen con líquidos isotónicos, en el período prodrómico o síndrome urémico hemolítico establecido, es segura y eficaz, reduce los requerimientos de diálisis, los días deinternación y de terapia intensiva, los eventos neurológicos y la hiponatremia.

Por ello, se propone, bajo supervisión nefrológica y/o garantizando el acceso a un centro de alta complejidad a corto plazo, hidratar a todo paciente sin signos de sobrecarga cardiopulmonar, independientemente de su función renal, con expansión inicial de volumen. Luego, si se logra una diuresis adecuada, no dializarlo (excepto que presente un trastorno metabólico/electrolítico intratable médicamente) y continuar la hidratación con una solución isotónica de dextrosa al $5 \%$ para una adecuada hidratación y diuresis.

Palabras clave: síndrome urémico hemolítico, deshidratación, fluidoterapia, líquidos extracelulares.

http: / / dx.doi.org/10.5546/ aap.2021.62

Texto completo en inglés:

http:/ / dx.doi.org/ 10.5546/ aap.2021.eng.62

a. Departamento de Urgencias.

b. Servicio de Nefrología.

Hospital de Niños

"Ricardo Gutiérrez" de Buenos Aires,

Argentina.

Correspondencia:

Dr. Manuel D. Bilkis: mbilkis@gmail.com

Financiación:

Ninguno.

Conflicto de intereses: Ninguno que declarar.

Recibido: $19-7-2020$

Aceptado: 15-9-2020
Cómo citar: Bilkis MD, Bonany P. Hidratación en el síndrome urémico hemolítico. Arch Argent Pediatr 2021;119(1):62-66.

\section{INTRODUCCIÓN}

Las enterobacterias productoras de toxina Shiga, en especial la E. coli (Shiga toxin-producing Escherichia coli; $S T E C$, por sus siglas en inglés), son las responsables de producir daño sistémico mediante microangiopatías trombóticas. Este compromiso puede afectar varios órganos, entre ellos, hígado, páncreas, piel, corazón, cerebro y, particularmente, los riñones. Sumado a la microangiopatía, la deshidratación y el menor flujo sanguíneo renal pueden empeorar el pronóstico de este cuadro, conocido como síndrome urémico hemolítico (SUH). ${ }^{1}$

El síndrome urémico hemolítico asociado a diarrea (SUHD+) se define por la presencia de un cuadro de gastroenteritis seguido por plaquetopenia (menos de 150000 plaquetas por $\mathrm{mm}^{3}$ ), anemia hemolítica microangiopática (hematocrito menor del $30 \%$ ) e insuficiencia renal aguda con valores de creatininemia por encima del límite superior para la edad. El SUHD+ carece de un tratamiento específico. En gran parte del mundo, la E. coli O157: H7 es la principal causa de SUHD+.

Las medidas de sostén están recomendadas como terapéutica, dentro de las que se incluye, tradicionalmente, la restricción hidroelectrolítica para evitar la sobrecarga cardiopulmonar de líquidos en la etapa aguda. ${ }^{2}$ Esta medida de restricción de volumen debería ser revisada por la correlación entre deshidratación, bajos aportes de líquidos isotónicos y mala evolución en el SUH.

\section{Síndrome urémico hemolítico y deshidratación}

La evolución desde la infección por STEC al SUH, que se da en un 10-15\% de los casos, era asumida como una fatalidad inmodificable. El SUH puede ser categorizado como oligoanúrico o no oligoanúrico. Los niños con SUH oligúricos, generalmente, requieren diálisis, tienen cursos más complicados y tienen mayor riesgo de secuelas crónicas. Sin embargo, se desconocía si los eventos que ocurrían temprano en las infecciones por STEC, en particular, la deshidratación y las medidas para expandir el volumen circulante, afectarían la probabilidad 
de una evolución a la oligoanuria y la insuficiencia renal aguda del SUHD+.

J. A. Ake y col., ${ }^{3}$ compararon, en forma retrospectiva, a 2 grupos de pacientes con SUHD+, uno oligoanúrico y otro no oligoanúrico, y observaron que el volumen y el contenido de sodio de los fluidos intravenosos administrados temprano en la enfermedad habían afectado el riesgo de desarrollar SUH oligoanúrico después de infecciones por E. coli O157: H7. Los pacientes con SUH oligoanúricos habían recibido menor aporte hídrico y de sodio al inicio de su enfermedad y habían presentado peores resultados evolutivos: mayor porcentaje de pacientes con necesidad de diálisis y de días de internación. Los autores propusieron que los pacientes con diarrea sanguinolenta (bloody diarrea; $B D$, por sus siglas en inglés) por $S T E C$ se beneficiarían expandiendo el espacio intravascular con soluciones isotónicas a razón de $20 \mathrm{ml} / \mathrm{kg}$ más un mantenimiento endovenoso según necesidades basales, antes del diagnóstico y desarrollo de SUHD+. ${ }^{3}$ C. A. Gianantonio y col., ${ }^{4}$ describieron un mejor pronóstico a largo plazo en pacientes con menor tiempo de oligoanuria inicial.

La deshidratación inicial en el SUHD+ predispone a mayor prevalencia de diálisis, como lo describieron Balestracci y col., ${ }^{5}$ quienes revisaron los datos de 137 niños con $\mathrm{SUH}$, que dividieron en dos grupos según su estado de hidratación al ingresar: normohidratados (n: 86) y deshidratados (n: 51). El grupo de pacientes deshidratados tuvo una mayor necesidad de diálisis (el 70,6\% versus el 40,7\%, p =0,0007). ${ }^{5}$ Concluyeron que la deshidratación en el ingreso hospitalario podría representar un factor concomitante que agravara la enfermedad renal intrínseca en pacientes con SUHD+. Y, por lo tanto, recomendaron el reconocimiento temprano de los pacientes con riesgo de SUHD+ para garantizarles un buen estado de hidratación con líquidos isotónicos.

J. M. Ojeda y col., ${ }^{6}$ estudiaron a 36 pacientes con SUHD+; 21 de ellos requirieron diálisis (el $58 \%$, intervalo de confianza-IC- del $95 \%$ : de 40,8 a 75,8$)$ y 13 (el $36,1 \%$; IC $95 \%$ : de 19,0 a 53,1$)$ no recuperaron por completo su función renal. En un modelo de análisis bivariado, solo fue un factor de riesgo significativo la deshidratación, definida como una pérdida de peso mayor del $5 \%$ (odds ratio $[O R]$ 5,3; IC $95 \%$ : de 1,4 a 12,3; $\mathrm{p}=0,0220)$. En un modelo multivariado (regresión de Cox), fue marginalmente significativa la deshidratación (cociente de riesgo: 95,823; IC $95 \%$ : de 93,175 a 109,948; $p=0,085)$. Los resultados obtenidos sugirieron que la deshidratación previa a la internación podía constituir un factor que incrementara el riesgo de presentar una recuperación incompleta de la función renal a largo plazo en niños que padecieron SUHD+.

En pacientes con SUHD+, Ardissino y col., ${ }^{7}$ estudiaron en forma retrospectiva la presencia de factores asociados con diálisis y/o compromiso neurológico. Este grupo planteó que la hemoconcentración y la hipovolemia, asociados a deshidratación, podían ser responsables de isquemia más grave y daño de órganos tanto a corto como a largo plazo. Estos signos, al inicio de la enfermedad, deben considerarse factores de riesgo de evolución más grave. Por lo tanto, recomendaron que el estado de hidratación debía ser monitoreado de modo activo en pacientes con SUH y que la deshidratación debía ser corregida puntualmente. $^{7}$

En un trabajo retrospectivo, multicéntrico y observacional, en nuestro medio, L. Alconcher y col.,${ }^{8}$ hallaron una mortalidad en SUHD+ del $3 \%$, la cual estuvo relacionada estadísticamente con hiponatremia, hemoconcentración y / o compromiso del sistema nervioso central (SNC). Otros autores describieron combinaciones de exámenes complementarios iniciales en el SUHD+ que definían la deshidratación como predictora de mala evolución. ${ }^{8-12}$

\section{Síndrome urémico hemolítico e hidratación}

La presentación a la atención médica de un niño con sospecha o confirmación de infección por STEC pero antes de que ocurra el SUH es una oportunidad potencial para mitigar el curso de la insuficiencia renal posterior. Hickey y col., ${ }^{13}$ estudiaron en forma prospectiva, multicéntrica y observacional los efectos de expandir con líquidos isotónicos en pacientes con diarrea por STEC previa al desarrollo del SUHD+. De los 50 participantes, el $68 \%$ estaba oligoanúrico. Llamativamente, dentro del grupo de los que no recibieron líquidos intravenosos (no expandidos) en los primeros 4 días de enfermedad, la tasa de oligoanúricos fue del $84 \%$. Los investigadores plantearon que la expansión del volumen (EV) intravenoso era una intervención infrautilizada que podría disminuir la frecuencia de insuficiencia renal oligoanúrica en pacientes con riesgo de $\mathrm{SUH} .{ }^{13}$

D. Loconsole y col., ${ }^{14}$ realizaron un protocolo operativo para la $B D$ en una población pediátrica 
como una respuesta rápida a una amenaza para la salud representada por un exceso de casos pediátricos de SUHD+ en la región de Apulia (Italia) a partir de 2013. Los casos positivos para STEC recibieron una vigorosa EV. El 7,5 \% desarrolló SUH, todos con evolución favorable.

R. McKee y col., ${ }^{15}$ en un estudio multicéntrico en 38 hospitales pediátricos de EE. UU. y Canadá, estudiaron a 927 niños infectados por STEC; 41 (el 4,4 \%) tenían SUH en el momento de la presentación; de los 886 restantes, 126 (el 14,2 \%) desarrollaron SUH. Los predictores de SUH incluyeron una edad más joven (OR 0,77; IC $95 \%$ : 0,69-0,85/año), recuento de leucocitos $\geq 13,0 \mathrm{x}$ $10^{3} / \mu \mathrm{l}$ (OR: 2,54; IC $95 \%$ : 1,42, 4,54), hematocrito más alto (OR: 1,83; IC $95 \%$ : 1,21, 2,77 / aumento del $5 \%$ ) y creatinina sérica (OR: 10,82; IC $95 \%$ : $1,49,78,69$ / aumento de $1 \mathrm{mg} / \mathrm{dl})$, recuento de plaquetas < $250 \times 10^{3} / \mu$ l (OR: IC $95 \%$ : 1,02, 3,60 ), menor sodio sérico (OR: 1,12; IC $95 \%$ : 1,02, 1,23/1 $\mathrm{mmol} / 1$ de disminución), y la administración de líquidos por vía intravenosa, que comenzó $\geq 4$ días después del inicio de la diarrea (OR: 2,50; IC 95 \%: 1,14, 5,46). Los factores de riesgo que identificaron destacaron la importancia de evitar la deshidratación mediante la EV temprana en la $B D$ y realizar un estrecho seguimiento clínico y de laboratorio.

G. Ardissino y col., ${ }^{16}$ hidrataron a los pacientes con líquidos isotónicos al debut del SUHD+ hasta conseguir un peso un $10 \%$ por encima del de referencia de normohidratación. El objetivo era restaurar el volumen circulante y reducir el daño renal por isquemia o hipoxia. Los resultados a corto y largo plazo de estos pacientes se compararon con un grupo de pacientes históricos en los que la terapéutica indicada era la restricción de líquidos. Los pacientes tuvieron resultados significativamente mejores a corto plazo, con una menor tasa de afectación del SNC (el 7,9 \% vs. el $23,7 \%, \mathrm{p}=0,06$ ), menor necesidad de diálisis (el $26,3 \%$ vs. el $57,9 \%, p=0,01)$ o días de cuidados intensivos ( 2,0 vs. 8,5 días, $p=0,02)$, y necesitaron menos días de hospitalización (9,0 vs. 12,0 días, $\mathrm{p}=0,03)$. A largo plazo, los resultados también fueron significativamente mejores en términos de secuelas renales y extrarenales (el 13,2\% vs. el 39,5 \%, p = 0,01). Así, este grupo concluyó que los pacientes con SUHD+ obtuvieron un gran beneficio de la EV temprana. Y sugirieron que las infusiones de líquidos tempranas y generosas podían reducir la formación de trombos y daño a los órganos isquémicos, lo que tendría efectos positivos en la enfermedad a corto y largo plazo. ${ }^{16}$
En pacientes adultos, se estudió un brote epidémico de SUHD+ en que se utilizó la EV como estrategia temprana previa al desarrollo del cuadro, en el que necesitaron diálisis el $30 \%$ de los casos $(5 / 15),{ }^{17}$ que fue menor que el $54 \%$ $(160 / 298)$ de otro brote en el que no usaron EV. ${ }^{18}$

Recientemente, en el Pediatric Nephrology, ${ }^{19}$ se publicó un trabajo retrospectivo nuestro en el que se analizaron treinta y cinco pacientes con SUHD+; 16 recibieron EV con líquidos isotónicos y 19 fueron pacientes que recibieron tratamiento convencional con restricción de fluidos (RF). Ninguno de los grupos presentó evidencia de sobrecarga hídrica al ingresar o durante el tratamiento. Se halló que solo el 12,5 \% de los pacientes del grupo de EV tuvo requerimiento de terapia de reemplazo contra el 47,4\% del grupo de RF. Además, la EV corrigió la hiponatremia inicial, y el sodio sérico se mantuvo dentro de los rangos normales.

Con respecto a las complicaciones neurológicas y la mortalidad, se encontró menos o ningún evento en el grupo de EV, aunque no mostraron diferencias estadísticas, probablemente, relacionado con el tamaño pequeño de la muestra. ${ }^{19}$ Por ello, se propone, inicialmente, bajo control nefrológico y/o, garantizándose el acceso a un centro de alta complejidad a corto plazo, hidratar a todo paciente con SUHD+ sin signos de sobrecarga de líquidos, independientemente del estado de la función renal, infundiendo solución salina al $0,9 \%$ a razón de $10 \mathrm{ml} / \mathrm{kg} /$ hora durante tres horas. Luego, si se logra una diuresis superior a $0,5 \mathrm{ml} / \mathrm{kg} /$ hora, no dializarlo (excepto que presente un trastorno metabólico o electrolítico intratable médicamente) y continuar la hidratación dependiendo de las necesidades del paciente, con una solución salina isotónica de dextrosa al $5 \%$ durante 48 horas, con el objetivo de mantener una adecuada hidratación y diuresis (Tabla 1).

\section{Expansión de volumen en el síndrome urémico hemolítico}

C. Ahn y col., ${ }^{20-22}$ abogan por la EV en la fase de $B D$ por enterobacterias productoras de toxina Shiga, primero con una expansión a razón de $20 \mathrm{ml} / \mathrm{kg}$ de solución fisiológica al 0,9\% y luego líquidos de mantenimiento con potasio, si este es normal o bajo. Se continúa esta conducta hasta la mejoría de los síntomas o el mantenimiento de las plaquetas por encima de $150000 / \mathrm{mm}^{3}$. Se requieren controles diarios de hemograma con recuento plaquetario, electrolitos, uremia y creatininemia. 
TABla 1. Protocolo de expansión de volumen

1) Criterios de inclusión: cada paciente con diagnóstico de SUHD+, normotenso, con una silueta cardíaca normal en radiografía de tórax y sin signos de sobrecarga de líquidos, independientemente de su función renal.

2) Dosis: infusión de $10 \mathrm{ml} / \mathrm{kg} /$ hora de solución de ClNa al 0,9\% durante un período de tres horas.

Posteriormente, si el paciente logró una diuresis superior a $0,5 \mathrm{ml} / \mathrm{kg} / \mathrm{hora}$, el tratamiento debe continuar con una solución salina isotónica (130-154 mEq/1) en dextrosa al $5 \%$ según necesidades basales de acuerdo con la fórmula de Holliday y Segar: de 0-10 kg, 100 ml/kg/día; de 10 a 20 kg, 50 ml/kg/día, y más de 20 kg, 20 ml/kg/día.

\section{S. Grisaru y col., ${ }^{23}$ realizaron una revisión} y metaanálisis acerca de la administración de líquidos endovenosos, estado de hidratación y evolución previa al desarrollo de SUHD+. Hallaron que un valor de hematocrito superior al $23 \%$ como medida del estado de deshidratación en la presentación con SUH se asoció con el desarrollo de SUH oligoanúrico (OR 2,38 [IC $95 \%: 1,30-4,35]$; I2 = $2 \%$ ), necesidad de terapia de reemplazo renal (OR 1,90 [IC $95 \%$ : 1,25-2,90]; I2 $=17 \%)$ y muerte $(O R$ 5,13 [IC $95 \%: 1,50$ $17,57] ; \mathrm{I} 2=55 \%$ ). La administración de líquidos endovenosos hasta el día del diagnóstico de SUH se asoció con una disminución del riesgo de insuficiencia renal y diálisis (OR 0,26 [IC $95 \%$ : $0,11-0,60]) .^{23}$

\section{Síndrome urémico hemolítico asociado a diarrea e hiponatremia}

La hiponatremia es una presentación inicial común de pacientes con diagnóstico de SUHD+ (entre el $30 \%$ y el $50 \%$ de los casos, según las series). Dos condiciones concomitantes son su raíz fisiopatológica. Primero, un fuerte estímulo no osmótico a la hormona antidiurética, como consecuencia de la deshidratación, vómitos, anemia, inflamación intestinal y dolor abdominal; $\mathrm{y}$, segundo, los intentos de hidratar a los pacientes mediante la administración oral de soluciones hipotónicas durante el período prodrómico, que es prolongado y progresivo. La hiponatremia está relacionada con complicaciones del SNC, como convulsiones y encefalopatía, y también es un fuerte predictor del riesgo de muerte en pacientes con SUHD+. ${ }^{24-27}$

En nuestra investigación, la hiponatremia inicial se corrigió en 24 horas, y la natremia se mantuvo dentro de los rangos normales en el grupo de EV. Después de 48 horas, el número de pacientes con hiponatremia fue estadística y significativamente mayor en el grupo de RF $(\mathrm{p}=0,014){ }^{19}$

\section{CONCLUSIONES}

La EV con líquidos isotónicos en los pacientes con diarrea por enterobacterias productoras de toxinas Shiga y al momento del diagnóstico de SUH establecido, es segura y eficaz. Mejora y mitiga la evolución del SUH.

\section{REFERENCIAS}

1. Gianantonio C, Vitacco M, Mendilaharzu F, Ruty A, et al. The hemolytic-uremic syndrome. J Pediatr. 1964; 64(4):478-91.

2. Grisaru S. Management of hemolytic-uremic syndrome in children. Int J Nephrol Renovasc Dis. 2014; 7:231-9.

3. AkeJA, JelacicS, CiolMA, WatkinsSL, etal. Relativenephroprotection during Escherichia coli O157:H7 infections: association with intravenous volume expansion. Pediatrics. 2005; 115(6):e673-80.

4. Gianantonio CA, Vitacco M, Mendilaharzu F, Gallo G. The hemolytic-uremic syndrome. Renal status o/76 patients at long-term follow-up. J Pediatr. 1968; 72(6):757-65.

5. Balestracci A, Martin SM, Toledo I, Alvarado C, et al. Dehydration at admission increased the need for dialysis in hemolytic uremic syndrome children. Pediatr Nephrol. 2012; 27(8):1407-10.

6. Ojeda JM, Kohout I, Cuestas E. La deshidratación al ingreso es un factor de riesgo para la recuperación incompleta de la función renal en niños con síndrome urémico hemolítico. Nefrología. 2013; 33(3):372-6.

7. Ardissino G, Daccò V, Testa S, Civitillo CF, et al. Hemoconcentration: a major risk factor for neurological involvement in hemolytic uremic syndrome. Pediatr Nephrol. 2015; 30(2):345-52.

8. Alconcher LF, Coccia PA, Suarez ADC, Monteverde ML, et al. Hyponatremia: a new predictor of mortality in patients with Shiga toxin-producing Escherichia coli hemolytic uremic syndrome. Pediatr Nephrol. 2018; 33(10):1791-8.

9. Ardissino G, Tel F, Testa S, Paglialonga F, et al. A simple prognostic index for Shigatoxin-related hemolytic uremic syndrome at onset: data from the ItalKid-HUS network. Eur J Pediatr. 2018; 177(11):1667-74.

10. Balestracci A, Meni Battaglia L, Toledo I, Martin SM, et al. Comment to: "A simple prognostic index for Shigatoxinrelated hemolytic uremic syndrome at onset: data from the ItalKid-HUS network" by Ardissino et al. Eur J Pediatr. 2018 Aug 10. doi: 10.1007 / s00431-018-3198-7. Eur J Pediatr. 2018; 177(12):1869-70.

11. Keenswijk W, Vanmassenhove J, Raes A, Dhont E, et al. Blood urea nitrogen to serum creatinine ratio is an accurate predictor of outcome in diarrhea-associated hemolytic uremic syndrome, a preliminary study. Eur J Pediatr. 2017; 176(3):355-60.

12. Balestracci A, Meni Battaglia L, Toledo I, Martin SM, et al. Blood urea nitrogen to serum creatinine ratio as a prognostic factor in diarrhea-associated hemolytic uremic syndrome: a validation study. Eur J Pediatr. 2018; 177(1):63-8.

13. Hickey CA, Beattie TJ, Cowieson J, Miyashita Y, et al. Early Volume Expansion During Diarrhea and Relative NephroprotectionDuringSubsequentHemolytic UremicSyndrome. 
Arch Pediatr Adolesc Med. 2011; 165(10):884-9.

14. Loconsole D, Giordano M, Laforgia N, Torres D, et al. Casemanagement protocol for bloody diarrhea as a model to reduce the clinical impact of Shiga toxin-producing Escherichia coli infections experience from Southern Italy. Eur J Clin Microbiol Infect Dis. 2020; 39(3):539-47.

15. McKee R, Schnadower D, Tarr P, Xie J, et al. Predicting Hemolytic Uremic Syndrome and renal replacement therapy in Shiga Toxin-producing Escherichia coli Infected children. Clin Infect Dis. 2020; 70(8):1643-51.

16. Ardissino G, Tel F, Possenti I, Testa S, et al. Early Volume Expansion and Outcomes of Hemolytic Uremic Syndrome. Pediatrics. 2016; 137(1):e20152153.

17. Sanders T, Ellis G, Castrovinci P, Deiss R, et al. 1098. Clinical Features and Outcomes of United States Marine Corps Recruits Hospitalized With Shiga Toxin-Producing Escherichia coli Infection and Hemolytic-UremicSyndrome. Open Forum Iinfect Dis. 2018; 5(Suppl 1):S329-30.

18. Menne J, Nistchke M, Stinngele R, Abu-Tair M, et al. Validation of treatment strategies for enterohaemorrhagic Escherichia coli O104:H4 induced haemolytic uraemic syndrome: case-control study. BMJ. 2012; 345:e4565.

19. Bonany P, Bilkis MD, Iglesias G, Braun A, et al. Fluid restriction versus volume expansion in children with diarrheaasociated HUS: a retrospective observational study. Pediatr Nephrol. 2020 Jul17. [Online ahead of print].

20. Ahn CK, Holt N, Tarr P. Shiga-Toxin Producing Escherichia coli and the Hemolytic Uremic Syndrome: What Have We Learned in the Past 25 Years? Adv Exp Med Biol. 2009; 634:1-17.

21. Holtz R, Neill M, Tarr P. Acute Bloody Diarrhea: A Medical Emergency for Patients of All Ages. Gastroenterology. 2009; 136(6):1887-98.

22. Davis TK, McKee R, Schnadower D, Tarr P. Treatment of Shiga Toxin-Producing Escherichia coli Infections. Infect Dis Clin North Am. 2013; 27(3):577-97.

23. Grisaru S, Xie J, Samuel S, Hartling L, et al. Associations Between Hydration Status, Intravenous Fluid Administration, and Outcomes of Patients Infected With Shiga Toxin-Producing Escherichia coli. A Systematic Review and Metaanalysis. JAMA Pediatr. 2017; 171(1):68-76.

24. Freedman S, Grisaru S, Xie J, Samuel Set al. Management of Shiga toxin producing Escherichia coli-infected children: A multi-national, multi-specialty survey.JPaediatr Child Health. 2018; 54(4):390-7.

25. Bilkis MD, Montero D, Vicente F, Cheistwer A. Hidratación endovenosa en la práctica clínica. Nuevos enfoques terapéuticos para la gastroenteritis aguda. Arch Argent Pediatr. 2007; 105(5):436-43.

26. Feld LG, Neuspiel DR, Foster BA, Leu MG, et al. Clinical Practice Guideline: Maintenance Intravenous Fluids in Children. Pediatrics. 2018; 142(6):e20183083.

27. Kamioka I, Yoshiya K, Satomura K, Kaito H, et al. Risk factors for developing severe clinical course in HUS patients: a national survey in Japan. Pediatr Int. 2008, 50(4):441-6.

\section{Artículos seleccionados}

Los siguientes resúmenes y comentarios de trabajos seleccionados se encuentran disponibles en la versión electrónica de este número.

N ENG J MED. 2020 Jul 2;383(1):49-57. DOI: 10.1056/NEJMoa1915075

Puntaje del Apgar y riesgo de muerte neonatal en recién nacidos prematuros (Cnattingius $S$, et al. Apgar score and risk of neonatal death among preterm infants)

Comentario: Dr. José M. Ceriani Cernadas. Editor Archivos Argentinos de Pediatría.

JAMA Pediatr. 2020 May 13. doi: 10.1001/ jamapediatrics.2020.1892.

Cierre de escuelas durante la pandemia de la enfermedad coronavirus 2019 (COVID). ¿Una intervención eficaz a nivel mundial? (Esposito S, et al. School closure during the coronavirus disease 2019 (COVID-19) pandemic: an effective intervention at the global level?)

Comentario: Dra. María Elina Serra. FUNDASAMIN-Fundación para la Salud Materno Infantil. Ciudad de Buenos Aires.

Arch Dis Child. 2020: 105: 253-9. doi:10.1136/ archdischild-2019-317561.

Oro líquido: costo-efectividad de los métodos de recolección de muestras de orina para niños pequeños precontinentes (Kaufman J, et al. Liquid gold: the cost-effectiveness of urine sample collection methods for young precontinent children)

Comentario: Dr. Gustavo Pereira. Hospital de Pediatría Dr. Juan P. Garrahan. Ciudad de Buenos Aires.

Pediatrics. October 2020;146 (4) e20201798; DOI: https: / / doi.org/10.1542 / peds.2020-1798

Seguimiento de potenciales epidemias de COVID-19 por las visitas de urgencia de casos con síntomas tipo influenza (Muchmore B, et al. Tracking potential COVID-19 outbreaks with influenza-like symptoms urgent care visits) Comentario: Dr. Norberto Giglio. Subcomisión de Investigación SAP. Hospital de Niños Ricardo Gutierrez. Ciudad de Buenos Aires.

Pediatrics. October 2020;146 (4):e2020005637

Resultados de las díadas de madres-recién nacidos después del SARS-CoV-2 materno (Verma S, et al. Outcomes of maternal-newborn dyads after maternal SARS-CoV-2)

Comentario: Dr. José M. Ceriani Cernadas. Editor Archivos Argentinos de Pediatría. 